\title{
Single- versus dual-attending strategy for spinal deformity surgery: 2-year experience and systematic review of the literature
}

\author{
*Ivan Cheng, MD, ${ }^{1}$ Martin N. Stienen, MD, FEBNS, ${ }^{2-4}$ Zachary A. Medress, MD, ${ }^{2}$ \\ Kunal Varshneya, BSc, ${ }^{2}$ Allen L. Ho, MD, ${ }^{2}$ John K. Ratliff, MD, ${ }^{2}$ and Anand Veeravagu, MD ${ }^{2}$ \\ Departments of ${ }^{1}$ Orthopedic Surgery and ${ }^{2}$ Neurosurgery, Stanford University Hospitals and Clinics, Stanford, California; \\ ${ }^{3}$ Department of Neurosurgery, University Hospital Zurich; and ${ }^{4}$ Clinical Neuroscience Center, University of Zurich, Switzerland
}

OBJECTIVE Adult spinal deformity (ASD) surgery is complex and associated with high morbidity and complication rates. There is growing evidence in the literature for the beneficial effects of an approach to surgery in which two attending physicians rather than a single attending physician perform surgery for and oversee the surgical care of a single patient in a dual-attending care model. The authors developed a dual-attending care collaboration in August 2017 in which a neurosurgeon and an orthopedic surgeon mutually operated on patients with ASD.

METHODS The authors recorded data for 2 years of experience with ASD patients operated on by dual attending surgeons. Analyses included estimated blood loss (EBL), transfusions, length of stay (LOS), discharge disposition, complication rates, emergency room visits and readmissions, subjective health status improvement, and disability (Oswestry Disability Index [ODI] score) and pain (visual analog scale [VAS] score) at last follow-up. In addition, the pertinent literature for dual-attending spinal deformity correction was systematically reviewed.

RESULTS The study group comprised 19 of 254 (7.5\%) consecutively operated patients who underwent thoracolumbar fusion during the period from January 2017 to June 2019 (68.4\% female; mean patient age 65.1 years, ODI score 44.5, VAS pain score 6.8). The study patients were matched by age, sex, anesthesia risk, BMI, smoking status, ODI score, VAS pain score, prior spine surgeries, and basic operative characteristics (type of interbody implants, instrumented segments, pelvic fixation) to 19 control patients (all $p>0.05$ ). There was a trend toward less EBL (mean $763 \mathrm{vs} 1524 \mathrm{ml}$, $p=0.059$ ), fewer intraoperative red blood cell transfusions (mean 0.5 vs 2.3, $p=0.079$ ), and fewer 90-day readmissions ( $0 \%$ vs $15.8 \%, p=0.071)$ in the dual-attending group. LOS and discharge disposition were similar, as were the rates of any < 30-day postsurgery complications, < 90-day postsurgery emergency room visits, and reoperations, and ODI and VAS pain scores at last follow-up (all $p>0.05$ ). At last follow-up, $94.7 \%$ vs $68.4 \%$ of patients in the dual- versus singleattending group stated their health status had improved $(p=0.036)$. In the authors' literature search of prior articles on spinal deformity correction, 5 of $8(62.5 \%)$ articles reported lower EBL and 6 of $8(75 \%)$ articles reported significantly lower operation duration in dual-attending cases. The literature contained differing results with regard to complication- or reoperation-sparing effects associated with dual-attending cases. Similar clinical outcomes of dual- versus singleattending cases were reported.

CONCLUSIONS Establishing a dual-attending care management platform for ASD correction was feasible at the authors' institution. Results of the use of a dual-attending strategy at the authors' institution were favorable. Positive safety and outcome profiles were found in articles on this topic identified by a systematic literature review.

https://thejns.org/doi/abs/10.3171/2020.3.SPINE2016

KEYWORDS adult spinal deformity; complications; deformity surgery; dual-attending strategy; estimated blood loss; outcome; spinal fusion; two-surgeon approach

\footnotetext{
ABBREVIATIONS AIS = adolescent idiopathic scoliosis; ALIF = anterior lumbar interbody fusion; $A S A=$ American Association of Anesthesiologists; $A$ SD = adult spinal deformity; DRG = Diagnosis Related Group; EBL = estimated blood loss; ER = emergency room; LLIF = lateral lumbar interbody fusion; LOS = length of stay; ODI = Oswestry Disability Index; PHQ-2 = Patient Health Questionnaire-2; PRBCs = packed red blood cells; PROM = patient-reported outcome measure; SNF = skilled nursing facility; SRS = Scoliosis Research Society; SVA = sagittal vertical axis; TLIF = transforaminal lumbar interbody fusion; VAS = visual analog scale.

ACCOMPANYING EDITORIAL See pp 557-559. DOI: 10.3171/2020.4.SPINE20465.
}

SUBMITTED January 4, 2020. ACCEPTED March 31, 2020.

INCLUDE WHEN CITING Published online July 10, 2020; DOI: 10.3171/2020.3.SPINE2016.

* I.C. and M.N.S. contributed equally to this work and share first authorship. 
A DULT spinal deformity (ASD) is a heterogenous condition characterized by varying degrees of pain and disability. Over the last century, care for ASD has evolved from supportive to corrective, resulting in new challenges to patients and spine surgeons alike. ${ }^{1}$ Surgical techniques have traditionally been based on instrumented fusion, with interbody supplementation and osteotomies utilized as needed. ${ }^{1}$ Despite the well-documented value of ASD correction in providing pain relief and restoring function, ${ }^{2}$ the high procedural complexity of this surgery, with $40 \%-70 \%$ complication and $30 \%$ reoperation rates, requires detailed informed consent, rigorous attempts at alternative management, and proceduralized operative environments. ${ }^{3,4}$

While spinal surgery for noncomplex conditions of the spine can safely be performed by trainees, ${ }^{5-9}$ this may not be true for spinal deformity correction procedures. Operative results and health-related quality of life after deformity surgery have been significantly and positively correlated with surgeon experience. ${ }^{10,11}$ Prior studies found shorter operating times and greater percentages of deformity correction with fellow compared with junior resident cosurgeons. ${ }^{11}$ More recently, a growing body of literature has included reports on dual-attending strategies for spinal deformity correction to improve patient safety. ${ }^{3,12-18}$

In an effort to optimize patient care while fostering collaboration between the neurosurgery and orthopedic departments, a neuro-ortho dual-attending collaboration was established at our institution for the evaluation, treatment, and follow-up of patients with challenging spinal conditions. Here we report our experience gained in the 2 years since the implementation of this strategy. In addition, we performed a systematic review of the literature, and we provide a summary of the currently available literature on complications and outcomes of dual- versus single-attending strategies for spinal deformity correction.

\section{Methods}

In August 2017, spine fellowship-trained authors I.C. and A.V. built a joint neuro-ortho spine service for the surgical treatment and pre- and postoperative evaluation of patients with ASD.

\section{Joint Neuro-Ortho Strategy}

The dual-attending approach to patients started in the outpatient clinic setting, where both surgeons aligned clinic days to evaluate patients together. This step was considered essential because $>60 \%$ of the patient population traveled $>2$ hours to be seen for evaluation. Patients who met the following criteria for neuro-ortho dual-attending care were those particularly prone to complications: patients with spinal deformity (cases of scoliosis, kyphosis, or flat-back syndrome; revision cases requiring at least 6 levels of fusion); older age; severe cardiac, pulmonary, or hemostatic comorbidities; a need for 3-column osteotomies; an expected long surgery time ( $>7$ hours); anticipated estimated blood loss (EBL) requiring transfusion; or an experienced surgeon deeming the surgery to be sufficiently complex..$^{19,20}$ After mutually deciding whether or not a patient required operative treatment, the two attending surgeons discussed and scheduled the type of surgery, approaches, and need for intraoperative technology (intraoperative imaging, robotic assistance, neuromonitoring, etc.). All surgical patients attended a 1.5-hour face-to-face interview with advanced practice providers that covered topics including the postoperative recovery period and allowed for a question-and-answer session. All patients were then engaged in a consent process that included a thorough discussion of risks, including bleeding, infection, proximal junctional kyphosis, rod/hardware failure, postoperative neurological injury, and medical complications such as stroke, venous thromboembolism, blindness, and death. ${ }^{21}$ On the operative day, both surgeons saw the patient before anesthesia administration and organized the setup and positioning with support by residents and/or fellows. Intraoperatively, both attending surgeons were viewed as equal members of the team, rather than having primary and secondary roles, and operated simultaneously and in parallel..$^{21}$ For postoperative care and until discharge, each patient was assigned to either the neurosurgical or the orthopedic acute care ward, but both surgeons attended to the patient during recovery and until discharge. The patients were again seen by both surgeons in postoperative outpatient follow-up-either mutually or sequentially.

The use of regular preoperative multidisciplinary case discussions in our dual-attending system is similar to the use of case discussions in a system previously reported by spinal surgeons in Seattle. ${ }^{21}$ Controversial management algorithms and complex cases were often presented for discussion with a wider team of spine surgeons at a monthly joint neuro-ortho conference.

\section{Identification of Study and Control Patients}

In a first step, electronic patient charts from our institution were reviewed to identify 681 patients who underwent surgery performed by either I.C. or A.V. since August 2017. Of those, 19 patients who underwent surgery performed mutually by the neurosurgeon and orthopedic surgeon attendings were identified as the "dual-attending group," and data for Diagnosis Related Group (DRG) codes were extracted for those patients.

We then essentially chose two ways to come up with control groups. All control patients were treated during the same overlapping time period (from August 2017 to June 2019) in order to exclude bias resulting from changes in perioperative care protocols, ever-increasing surgeon experience and efficiency, and improvements in techniques and general system-wide improvements.

First, we excluded 427 patients with DRG codes that deviated from those of the patients in the dual-attending group. The remaining 235 nonmatched single-attending patients from both I.C. and A.V. with similar DRG codes were compared to the 19 dual-attending cases (Fig. 1) with regard to some basic quality outcomes (Table 1). Although the advantage of this control group was that the patients were treated by the same two surgeons (either combined or individually), the disadvantage was that a selection was inevitably made with regard to case complexity. Therefore, in a second step, we identified 19 controls 


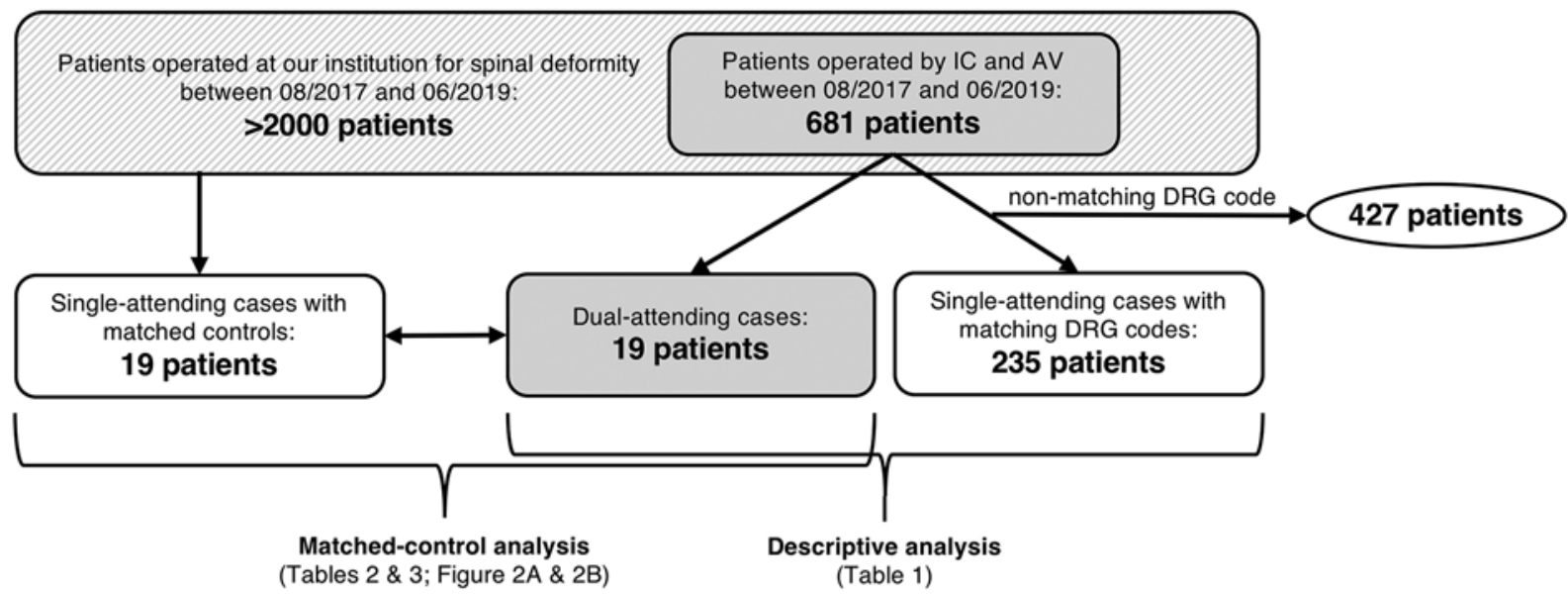

FIG. 1. Illustration of how the study group ( $n=19$ dual-attending cases) and the two single-attending control groups $(n=19$ matched controls or $n=235 D R G$ code-matched controls) were composed from the total population of spine patients treated at our institution during the period from August 8, 2017, to June 6, 2019.

matched for age, sex, surgical procedure, and more detailed parameters from the cohort of spine patients treated during the overlapping time period by a single attending surgeon performing spinal deformity surgery at our institution (Table 2). Because both surgeons engaging in the neuro-ortho dual-attending collaboration performed their complex cases together, only 2 matched patients (in surgical cases for which the other cosurgeon was unavailable) were operated on by those two contributing surgeons, and the remaining cases for matching were patients who underwent surgery performed by other spine surgery attendings.

\section{Data Collection and Variable Description}

The following demographic variables were extracted from the electronic patient charts: age at time of index surgery (in years), sex, anesthesia risk (American Association of Anesthesiologists [ASA] score), BMI (in $\mathrm{kg} / \mathrm{m}^{2}$ ), and smoking status at time of surgery (never smoker, active smoker, or former smoker with at least 2 months of smoking cessation). ${ }^{22}$

Disease-specific variables were patient-reported outcome measures (PROMs) at the last pre- and last postoperative clinic encounters, including the Oswestry Disability Index (ODI) score ranging from 0 (best) to 100 (worst), visual analog scale (VAS) score for average daily back and leg pain, and Patient Health Questionnaire-2 (PHQ-2) score ranging from 0 (best) to 6 (worst). Depression was defined as a PHQ-2 score $\geq 3 .{ }^{23}$ We recorded the number of previous spine surgeries; the presence of sagittal and/ or coronal imbalance; the number of instrumented spinal/ pelvic levels; the number of levels with anterior lumbar interbody fusion (ALIF), lateral lumbar interbody fusion (LLIF), or transforaminal lumbar interbody fusion (TLIF); and pelvic fixation using either iliac screws or S2alar-iliac screw trajectories.

In addition to the PROMs, our outcomes of interest included EBL (in $\mathrm{ml}$ ), number of units of intraoperative transfusion with packed red blood cells (PRBCs; decided
TABLE 1. Basic features of single- versus dual-attending cases

\begin{tabular}{lccr}
\hline \multicolumn{1}{c}{ Variable } & $\begin{array}{c}\text { Dual Attending } \\
(\mathrm{n}=19)\end{array}$ & $\begin{array}{c}\text { Single Attending } \\
(\mathrm{n}=235)\end{array}$ & $\mathrm{p}$ Value \\
\hline DRG code & & & 0.101 \\
\hline MS453 & $1(5.3 \%)$ & $7(3.0 \%)$ & \\
\hline MS454 & $13(68.4 \%)$ & $92(39.2 \%)$ & \\
\hline MS455 & $2(10.5 \%)$ & $68(28.9 \%)$ & \\
\hline MS457 & $1(5.3 \%)$ & $9(3.8 \%)$ & \\
\hline MS460 & $2(10.5 \%)$ & $59(25.1 \%)$ & \\
\hline LOS, days & $6.4(5.0-7.7)$ & $4.1(3.8-4.4)$ & $<0.001$ \\
\hline Discharge disposition & & & 0.339 \\
\hline Home & $10(52.6 \%)$ & $158(67.2 \%)$ & \\
\hline Rehab/SNF & $9(47.4 \%)$ & $74(31.5 \%)$ & \\
\hline Other hospital & $0(0.0 \%)$ & $3(1.3 \%)$ & \\
\hline Any complication w/in & & & \\
$<30$ days & & & \\
\hline No & $17(89.5 \%)$ & $211(89.8 \%)$ & \\
\hline Yes & $2(10.5 \%)$ & $24(10.2 \%)$ & \\
\hline$<90$-day readmission & & & 0.757 \\
\hline No & $19(100 \%)$ & $226(96.2 \%)$ & \\
\hline Yes & $0(0.0 \%)$ & $9(3.8 \%)$ & \\
\hline$<90$-day ER visit & & & \\
\hline No & $18(94.7 \%)$ & $226(96.2 \%)$ & \\
\hline Yes & $1(5.3 \%)$ & $9(3.8 \%)$ & \\
\hline MS453 & & & \\
\hline
\end{tabular}

MS453 = combined anterior/posterior spinal fusion with major complication or comorbidity (MCC); MS454 = combined anterior/posterior spinal fusion with complication or comorbidity (CC); MS455 = combined anterior/posterior spinal fusion without CC/MCC; MS457 = spinal fusion except cervical with spinal curvature or malignancy or infection or extensive fusions with CC; MS460 = spinal fusion except cervical without MCC.

Values are presented as number of patients (\%) or median (IQR).

* Complications included postoperative anemia requiring transfusion, myocardial infarction, pneumonia, congestive heart failure, sepsis, deep venous thrombosis, pulmonary embolism, respiratory failure, surgical site infection, or ileus within $<30$ postoperative days. 
TABLE 2. Basic information about the cohort of joint neuro-ortho patients and a matched cohort of patients treated by a single neurosurgery or orthopedic surgery attending

\begin{tabular}{|c|c|c|c|}
\hline & $\begin{array}{l}\text { Single Attending } \\
\qquad(n=19)\end{array}$ & $\begin{array}{l}\text { Joint Neuro-Ortho } \\
\qquad(\mathrm{n}=19)\end{array}$ & $\mathrm{p}$ Value \\
\hline Age, yrs & $70.1(66.1-74.0)$ & $65.1(59.3-70.8)$ & 0.143 \\
\hline Sex & & & 0.189 \\
\hline Female & $9(47.4 \%)$ & $13(68.4 \%)$ & \\
\hline Male & $10(52.6 \%)$ & $6(31.6 \%)$ & \\
\hline ASA score & & & 0.385 \\
\hline 2 & $6(31.6 \%)$ & $9(47.4 \%)$ & \\
\hline 3 & $13(68.4 \%)$ & $10(52.6 \%)$ & \\
\hline $\mathrm{BMI}, \mathrm{kg} / \mathrm{m}^{2}$ & $28.9(26.8-31.1)$ & $28.5(26.1-30.9)$ & 0.784 \\
\hline Smoking status & & & 0.088 \\
\hline Never & $10(52.6 \%)$ & $5(26.3 \%)$ & \\
\hline Active & $0(0 \%)$ & $3(15.8 \%)$ & \\
\hline Former & $9(47.4 \%)$ & $11(57.9 \%)$ & \\
\hline Preop depression* & & & 0.445 \\
\hline No & $15(78.9 \%)$ & $12(63.2 \%)$ & \\
\hline Yes & $2(10.5 \%)$ & $5(26.3 \%)$ & \\
\hline NA & $2(10.5 \%)$ & $2(10.5 \%)$ & \\
\hline ODI & $52.7(45.2-60.2)$ & $44.5(37.7-51.2)$ & 0.093 \\
\hline VAS pain & $6.5(5.6-7.4)$ & $6.8(6.0-7.6)$ & 0.583 \\
\hline Previous spine ops & $2(0-4)$ & $1(0-2)$ & 0.698 \\
\hline \multicolumn{4}{|l|}{ Preop imbalance } \\
\hline Sagittal plane & $15(79.0 \%)$ & $15(79.0 \%)$ & $>0.99$ \\
\hline Coronal plane & $4(21.1 \%)$ & $9(47.4 \%)$ & 0.087 \\
\hline Instrumented levels & $7.6(5.8-9.4)$ & $6.8(5.1-8.5)$ & 0.477 \\
\hline ALIF levels & & & 0.345 \\
\hline None & $10(52.6 \%)$ & $11(57.9 \%)$ & \\
\hline 1 & $2(10.5 \%)$ & $5(26.3 \%)$ & \\
\hline$\geq 2$ & $7(36.9 \%)$ & $3(15.8 \%)$ & \\
\hline LLIF levels & & & 0.197 \\
\hline None & $12(63.2 \%)$ & $5(26.3 \%)$ & \\
\hline 1 & $3(15.8 \%)$ & $4(21.0 \%)$ & \\
\hline$\geq 2$ & $4(21.1 \%)$ & $10(52.7 \%)$ & \\
\hline TLIF levels & & & 0.196 \\
\hline None & $16(84.2 \%)$ & $19(100 \%)$ & \\
\hline 1 & $2(10.5 \%)$ & $0(0 \%)$ & \\
\hline$\geq 2$ & $1(5.3 \%)$ & $0(0 \%)$ & \\
\hline Pelvic fixation $†$ & & & 0.328 \\
\hline No & $9(47.4 \%)$ & $12(63.2 \%)$ & \\
\hline Yes & $10(52.6 \%)$ & $7(36.8 \%)$ & \\
\hline
\end{tabular}

$\mathrm{NA}=$ not available

Data are presented as number of patients (\%) or median (IQR)

* Diagnosis based on preoperative PHQ-2 score $>3$ points.

† lliac bolt or S2-alar-iliac screw fixation.

by the attending anesthesiologist according to a standardized spine-specific transfusion protocol) or fresh-frozen plasma, main hospital length of stay (LOS; in days), and discharge disposition (home [with home health service], inpatient rehabilitation, or skilled nursing facility [SNF], or transfer to other acute care hospital). Furthermore, we recorded complications occurring $<30$ days postoperatively (postoperative anemia requiring transfusion, myocardial infarction, pneumonia, congestive heart failure, sepsis, deep venous thrombosis, pulmonary embolism, respiratory failure, surgical site infection, or ileus), emergency room (ER) visits within $<90$ days postsurgery, hospital readmissions within $<90$ days, and reoperations (including reasons) until last follow-up. Finally, at last clinic follow-up, the change of each patient's functional status was determined as improved, similar, or worsened compared to baseline. ${ }^{6,7}$

\section{Statistical Approach}

Descriptive statistics were used to compare the study group (19 dual-attending cases) to the single-attending control cases (235 DRG code-matched or 19 control cases). Chi-square tests and t-tests were used for group comparisons, and $\mathrm{p}$ values $<0.05$ were considered statistically significant.

\section{Literature Review}

The PRISMA guidelines were followed for conducting this systematic review. ${ }^{24}$ We searched the PubMed, Web of Science, EMBASE, and SCOPUS databases for permutations of the words "two," "attending," "dual," "surgeon," "spine," and "surgery" (see Appendix for search terms). We included all English, French, and German articles on human subjects with spinal deformity that reported on comparative outcomes of single- versus dual-attending strategies, published until July 2019. Full-text papers for which the title and abstract met predefined eligibility criteria were rigorously assessed to determine inclusion eligibility. References from each full-text article were similarly reviewed for inclusion eligibility. The study screening and data extraction were independently performed by M.N.S. and K.V. All relevant data (author, year, type of study, population, number of patients, EBL, operative time, complications, reoperations, clinical outcomes) were extracted from the articles; no contact with the authors of the included articles was required. Any discrepancies were resolved by a discussion between M.N.S. and K.V. or with the entire research group. Descriptive statistics were chosen. Risk of bias was not assessed. The review protocol was not prepublished or registered.

\section{Results}

\section{Unmatched Analysis}

Nineteen of 254 consecutive patients who underwent thoracolumbar fusion surgery performed by both I.C. and A.V. during the period from August 2017 to June 2019 were dual-attending cases (7.5\%; Fig. 1).

Compared with cases in which patients underwent surgery performed by I.C. or A.V. individually, the dualattending cases had a 2.3-day longer LOS ( $<<0.001)$, whereas discharge dispositions, < 30-day postoperative complications, and $<90$-day postoperative ER visits and readmissions were similar in the two groups (all $\mathrm{p}>0.05$; Table 1). 
TABLE 3. Comparison of outcomes of patients in the neuro-ortho dual-attending and single-attending groups

\begin{tabular}{|c|c|c|c|}
\hline & \multicolumn{2}{|c|}{ Patient Group } & \multirow[b]{2}{*}{$p$ Value } \\
\hline & Single Attending $(n=19)$ & Dual Attending $(n=19)$ & \\
\hline $\mathrm{EBL}, \mathrm{ml}$ & $1524(768-2280)$ & $763(441-1085)$ & 0.059 \\
\hline \multicolumn{4}{|l|}{ Intraop transfusion, no. of units } \\
\hline PRBCs & $2.3(0.3-4.3)$ & $0.5(0.0-1.0)$ & 0.079 \\
\hline FFP & $1.6(-0.2$ to 3.5$)$ & $0.3(-0.1$ to 0.7$)$ & 0.132 \\
\hline LOS, days & $6.3(4.8-7.8)$ & $6.4(5.0-7.7)$ & 0.957 \\
\hline Discharge disposition & & & 0.189 \\
\hline Home & $6(31.6 \%)$ & $10(52.6 \%)$ & \\
\hline Rehab/SNF & $13(68.4 \%)$ & $9(47.4 \%)$ & \\
\hline Any complication <30 days* & & & 0.374 \\
\hline No & $15(78.9 \%)$ & $17(89.5 \%)$ & \\
\hline Yes & $4(21.1 \%)$ & $2(10.5 \%)$ & \\
\hline 90-day readmission & & & 0.071 \\
\hline No & $16(84.2 \%)$ & $19(100 \%)$ & \\
\hline Yes & $3(15.8 \%)$ & $0(0 \%)$ & \\
\hline 90-day ER visit & & & 0.150 \\
\hline No & $15(78.9 \%)$ & $18(94.7 \%)$ & \\
\hline Yes & $4(21.1 \%)$ & $1(5.3 \%)$ & \\
\hline FU, days & $210(138-283)$ & $305(191-420)$ & 0.155 \\
\hline Reop before last FU & & & 0.676 \\
\hline No & $16(84.2 \%)$ & $15(79.0 \%)$ & \\
\hline Yes & $3(15.8 \%)$ & $4(21.0 \%)$ & \\
\hline Hardware protrusion & $0(0 \%)$ & $2(10.5 \%)$ & \\
\hline Pseudarthrosis & $0(0 \%)$ & $2(10.5 \%)$ & \\
\hline Wound infection & $2(10.5 \%)$ & $0(0 \%)$ & \\
\hline Repair of CSF leak & $1(5.3 \%)$ & $0(0 \%)$ & \\
\hline ODI score at last FU & $34.2(25.4-43.0)$ & $29.2(21.7-36.8)$ & 0.366 \\
\hline VAS pain score at last FU & $3.4(2.3-4.4)$ & $2.5(1.9-3.1)$ & 0.139 \\
\hline Postop change in status $†$ & & & 0.036 \\
\hline Improved & $13(68.4 \%)$ & $18(94.7 \%)$ & \\
\hline Similar & $6(31.6 \%)$ & $1(5.3 \%)$ & \\
\hline Worse & $0(0 \%)$ & $0(0 \%)$ & \\
\hline \multicolumn{4}{|c|}{$\begin{array}{l}\text { FFP = fresh-frozen plasma; FU = follow-up. } \\
\text { Data are presented as number of patients (\%) or median (IQR). } \\
{ }^{*} \text { Complications included postoperative anemia requiring transfusion, myocardial infarction, pneumonia, congestive } \\
\text { heart failure, sepsis, deep venous thrombosis, pulmonary embolism, respiratory failure, surgical site infection, or ileus } \\
\text { at }<30 \text { postoperative days. } \\
\dagger \text { Compared to preoperative, according to patient statement at last outpatient clinic visit. }\end{array}$} \\
\hline
\end{tabular}

\section{Matched-Cohort Analysis}

Both demographic patient characteristics and technical aspects of the surgery were comparable for the matched single- and dual-attending cohorts (all $\mathrm{p}>0.05$; Table 2).

Compared with the single-attending control cohort, the dual-attending cases had trends toward lower EBL ( $p=$ $0.059)$, less need for intraoperative PRBC transfusions ( $\mathrm{p}=$ 0.079 ), and fewer $<90$-day readmissions ( $\mathrm{p}=0.071$; Table 3 ). LOS was comparable between the groups, as were the rates of $<30$-day complications and $<90$-day ER visits. Over a mean follow-up of 210 and 305 days, reoperation rates were $15.8 \%$ in single- and $21.0 \%$ in dual-attending cases, respectively $(\mathrm{p}=0.676)$.

At the final follow-up in both the single- and dual-attending groups, the surgery was found to have been effective in decreasing pain (single attending: VAS difference 3.0, $\mathrm{p}<0.001$; dual attending: VAS difference 4.3, $\mathrm{p}$ $<0.001$ ) and disability (single attending: ODI difference 17.9, $\mathrm{p}<0.001$; dual attending: ODI difference 15.2, $\mathrm{p}=$ 0.001; Fig. 2A and B). At the final follow-up, patients in the dual-attending group were more likely to rate their functional status as improved ( $\mathrm{p}=0.036$; Table 3$)$. 


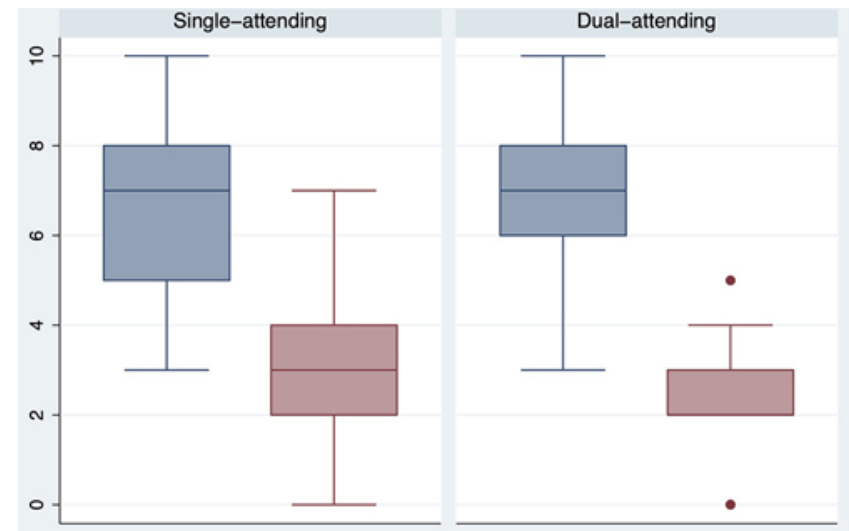

A

preoperative

postoperative
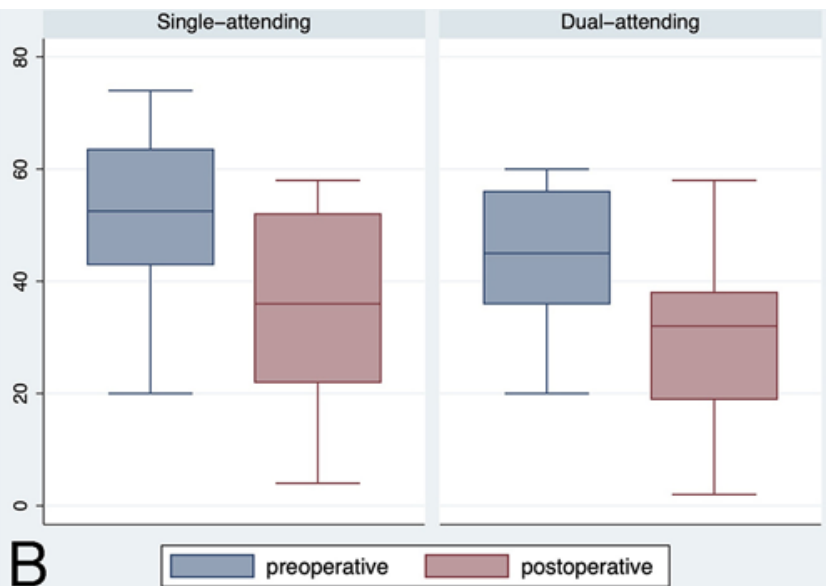

FIG. 2. Median preoperative (blue boxes) and postoperative (pink boxes) VAS pain scores (A) and ODI scores (B) with the 25th75th percentiles (boxes), the upper and lower adjacent values (whiskers), and outliers (dots) of patients in the single- or dualattending groups. Figure is available in color online only.

\section{Illustrative Case}

A 55-year-old man presented to our institution with a history of severe axial low-back pain and right lower-extremity pain after undergoing an attempted L2-S1 fusion at an outside institution. A CT myelogram showed evidence of pseudarthroses at multiple levels, loss of lumbar lordosis, and migration of multiple interbody implants into the spinal canal (Fig. 3). Standing radiographs showed a severe kyphotic deformity with a positive sagittal vertical axis (SVA). Given the extensive reconstruction required to remove the patient's previous hardware and correct his deformity, in addition to his complex medical comorbidities, we elected to implement a dual-attending approach in this case. Our operation consisted of removal of the patient's prior instrumentation and placement of T10-ilium posterior instrumented fusion with interbody placement at L4-5 and L5-S1 and deformity correction, including posterior column osteotomies. The patient developed an ileus postoperatively, which he recovered from, and otherwise did very well and was discharged to a nursing facility on
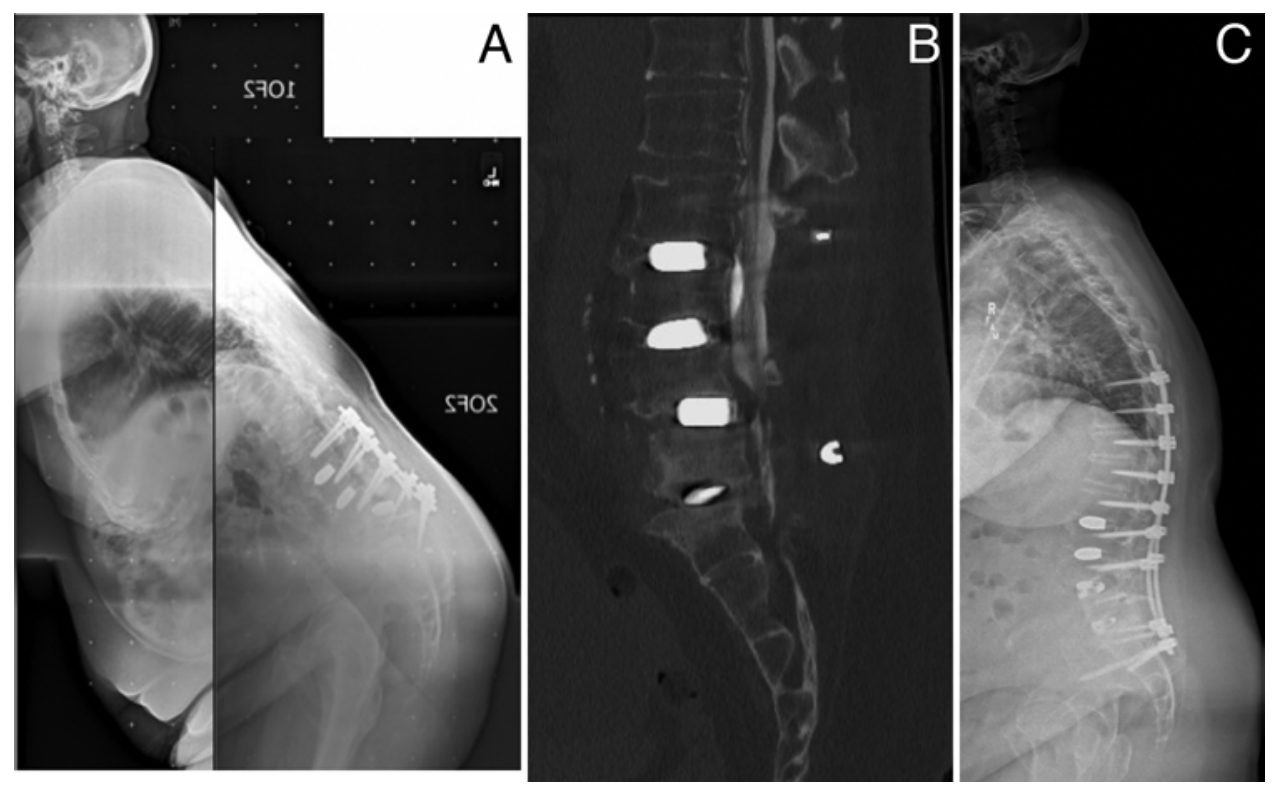

FIG. 3. Illustrative case of a 55-year-old man who presented following attempted L2-S1 fusion at an outside institution. A: Standing radiographs showing a severe kyphotic deformity with a positive SVA. B: CT myelogram showing evidence of pseudarthroses at multiple levels, loss of lumbar lordosis, and migration of multiple interbody implants into the spinal canal. A dual-attending model was used in this case given its complexity and the patient's numerous comorbid medical conditions. C: Following T10-ilium posterior instrumented fusion with interbody placement at L4-5 and L5-S1 and deformity correction, postoperative standing radiographs showed correction of the patient's kyphotic deformity. 


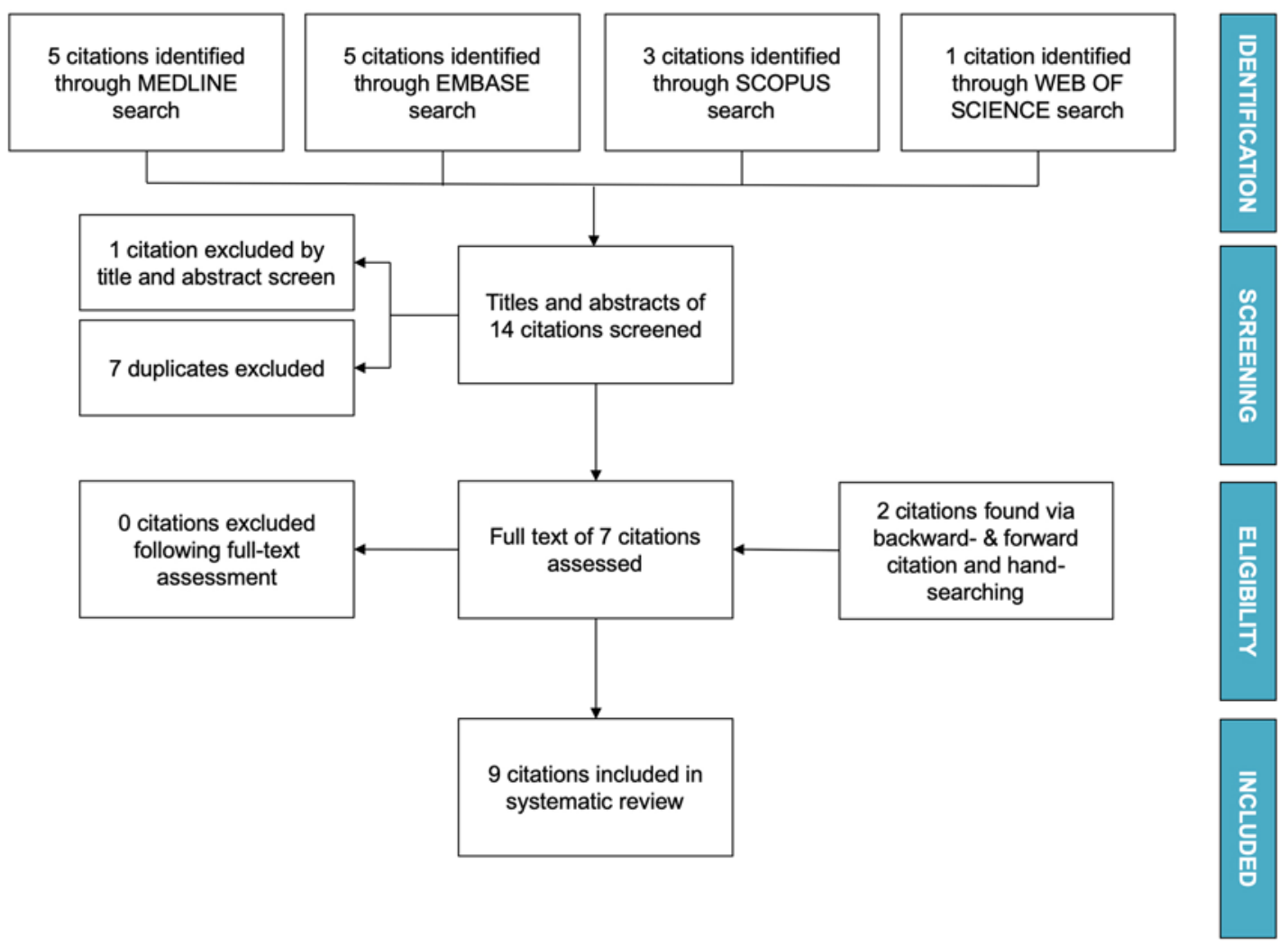

FIG. 4. PRISMA flowchart detailing the process for the selection of papers. Figure is available in color online only.

postoperative day 8 . Postoperative standing radiographs showed satisfactory correction of the patient's kyphotic deformity.

\section{Systematic Literature Review}

The 9 articles identified by the systematic literature review (Fig. 4) are listed in Table 4., ${ }^{3,12-18,21}$ Four were prospective and 5 were retrospective studies. Single- versus dual-attending strategies for adult deformity were reported on in 3 papers ${ }^{3,14,21}$ and for pediatric patients with adolescent idiopathic scoliosis (AIS), neuromuscular, or other forms of scoliosis surgery were reported on in 6 papers. ${ }^{12,13}$, 15-18 Three of the identified articles were derived from the same authors and institution, and there appeared to be some overlap in the reported data of those 3 articles..$^{13,16,17}$

Five of $8(62.5 \%)$ studies found that EBL was significantly lower in dual-attending cases, $, 3,13,16-18$ and 3 studies did not find a difference..$^{12,14,15}$ Six of $8(75 \%)$ studies found that operative time was significantly reduced, ${ }^{3,13,15-18}$ and 2 studies did not find a difference. ${ }^{12,14}$ Reporting of complications was handled very heterogeneously across included studies with regard to their definition and timing. Three studies found a lower need for PRBC transfusions in dualattending cases. ${ }^{13,16,17}$ One large multicenter study found fewer intraoperative complications, but a similar rate of (early) perioperative and a higher rate of (late) postoperative complications, in dual-attending cases. ${ }^{14}$ With regard to postoperative complications, 2 studies reported a significantly lower rate with dual surgeons (16\% vs $52 \%$ up to 90 days, $8 \%$ vs $33 \%$ up to 2 years), ${ }^{18,21}$ while 3 studies did not find any difference in complications between the groups (over differing time periods). ${ }^{3,12,15}$ Unplanned reoperation rates were reported for 3 prior studies, ${ }^{3,14}$ of which each report was in favor of the dual-attending group ${ }^{21} 1$ study reported similar rates and 1 study found significantly higher rates in the dual-attending group. Only 1 prior study reported clinical (PROM) outcomes and found comparable improvement on the Scoliosis Research Society (SRS) Outcomes Questionnaire (SRS-22), ODI, and SF-12 scores for both groups at 6 weeks and at 1 and 2 years. ${ }^{14}$

\section{Discussion}

There have been several reports about the beneficial effects of a dual-attending care plan at selected spinal deformity centers, including time savings in the operating room, shortened postoperative stay, and decreased complications, readmissions, and reoperations., ${ }^{3,12-18,21}$ A survey among 199 deformity surgeons from the SRS revealed that the majority believe that having two attending spine surgeons leads to improved safety, decreased complications, and improved outcomes compared with having one attending for deformity cases in general, as well as for 3-column osteotomy and other complex cases. ${ }^{20}$ However, the survey results also showed that most respondents do not often use a second attending, which reveals that there may be impediments to implementing dual-attending strategies into practice. Here, we report our experiences gained during 


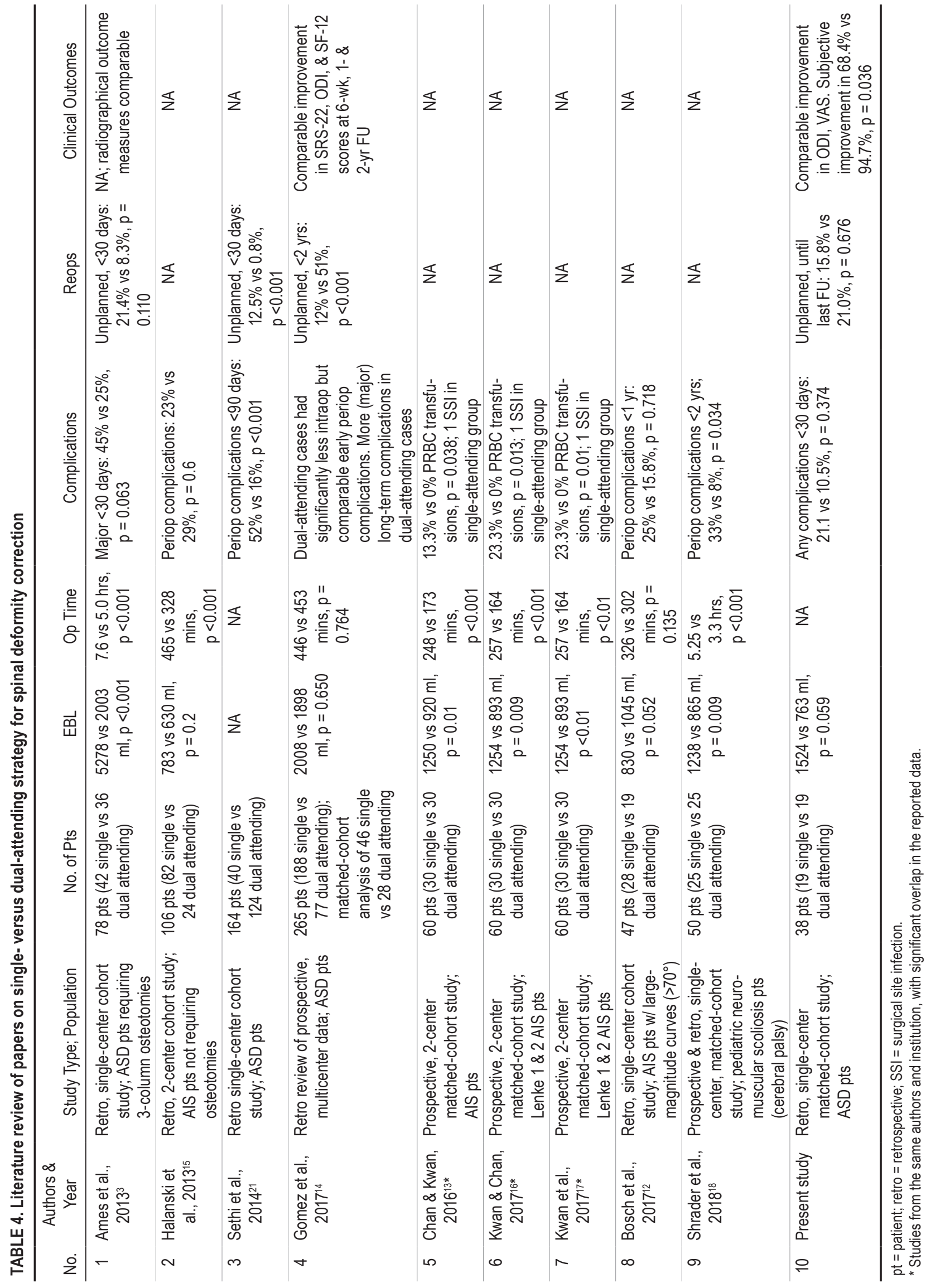


the previous 2 years after the establishment of a neuroortho dual-attending endeavor for the surgical treatment of patients with ASD.

\section{Feasibility, Reimbursement, and Cost-Effectiveness}

Several aspects are unique to the dual-attending approach and crucial to its feasibility. One is the importance of the neurosurgeon and orthopedic surgeon sharing similar practice philosophies regarding decision-making and surgical technique. In this situation, improved teamwork and precise cooperation can lead to demonstrable improvements in patient care. ${ }^{18}$ It is critical to maintain similar approaches to spinal parameters and surgical practices, and techniques such as approaches to anterior column reconstruction. Such surgical harmony will aid in improving outcomes. A second concern is addressing the time constraints for two busy spine surgeons, which can make allocating joint outpatient clinic and operating room time challenging. A third challenge is posed by the difficulties in determining the second surgeon's reimbursement, which has been identified as another key hindrance to the broader implementation of dual-attending strategies at most deformity centers. ${ }^{20}$

Currently, two attending surgeons of the same specialty can work together in the United States, but the second surgeon can only bill as an assistant, a position that carries significantly decreased work relative value units (wRVU). Surgeons of two different specialties can work together as cosurgeons for a modest increase in the total wRVU. Those aspects of feasibility have recently been discussed in depth in a report that includes a description of the Seattle approach, for which equal payment is given to both surgeons. ${ }^{19}$

In a DRG-based system, adding a cosurgeon does result in additional professional fees. In the United States, cosurgeons can each charge $62.5 \%$ of a content provider code, resulting in $125 \%$ of the charges that a single attending surgeon could bill for. However, the potential for reduced operative length and decreased rates of transfusions, and improvements in other quality metrics, could offset these added professional fees in a healthcare system, particularly in a bundled payment system, which is inherently structured to reward high-quality and well-coordinated patient care.

Costs of ASD surgery can be exorbitant, and large-scale analyses looking at long-term quality of care and cost-effectiveness are rare. Some data suggest that ASD surgery is cost-effective after a 10 -year period, ${ }^{25}$ and having two cosurgeons perform the procedure will likely increase the cost of operative treatment. ${ }^{14}$ However, recent attempts to decrease the costs of care include targeted efforts to avoid operative complications. Considering that dual-attending surgeries have been reported to be associated with reductions in (major) complications - as was evident in the findings of our literature review (Table 4) - this strategy may reduce costs in the long run..$^{15,19}$ Although a cost analysis was not performed as part of this study, the use of a twosurgeon team undoubtedly has financial implications. ${ }^{19} \mathrm{We}$ anticipate the need for future cost-effectiveness analyses in order to critically question whether the benefits of a dual-attending strategy outweigh the additional expenses.

\section{Patient Safety and Outcomes}

Multiple studies have consistently shown that spine surgery complications increase with increased operative duration, EBL, and necessity for transfusions, as well as with particularly challenging procedures, including deformity corrections requiring 3-column osteotomies. $3,4,12,26$ EBL in corrective spinal deformity surgery can exceed a patient's baseline total blood volume. ${ }^{21}$ Surgeon error or misjudgment is another reason behind potentially preventable complications in deformity surgery. ${ }^{21}$ Standardized protocols for high-risk spine surgery can help lower rates of intra- and postoperative complications, and surgeons face the need to develop strategies to increase patient safety at their institutions. Having two experienced surgeons simultaneously exposing, placing instrumentation, decompressing, performing the osteotomies, and closing the wounds can save significant time at each stage..$^{15,19}$ Correspondingly, the literature supports the notion that a dual-attending strategy leads to shorter case durations, $, 1,7,9$, 13,14,18 reduces EBL, , ,13,15-18 and decreases the necessity for transfusion, ${ }^{13,16,17}$ which may translate into greater patient safety and optimized outcomes. ${ }^{3,12-18,21}$ These effects have been found in both ASD cases ${ }^{3,14,21}$ and AIS cases ${ }^{13,15-17}$ that involved younger, healthier patients. ${ }^{19}$

The data show not only that the rates of surgical complications are reduced in dual-attending compared with single-attending cases-likely as a result of decreased operative times-but also that the rates of medical complications were lower in dual-attending cases, such as deep vein thrombosis and pulmonary embolism (10\% vs $3.2 \%)$ and urinary tract infections (32.5\% vs 9.7\%). ${ }^{21}$ Experts in the field have acknowledged the positive aspects of a dualattending strategy: $67.5 \%$ of the responding SRS surgeons indicated they would use a second attending if possible more often or always for deformity cases. ${ }^{24}$

In our series, the effects of a dual-attending approach may have been insignificant and mitigated for two reasons, namely the small sample size and the fact that experienced fellows often scrubbed to assist in the matched single-attending cases. We assume that the perception of better health reported by patients at follow-up (Table 3 ) might have been a result of the mutual follow-up strategy, for which patients have higher confidence in the quality of their healthcare when seeing two spine specialists in clinics postoperatively. Furthermore, the longer LOS (reported in Table 1) was likely due to greater case complexity in dual-attending compared with single-attending cases.

\section{Benefits for the Attendings}

True expertise is increasingly understood to include appropriate recognition of and response to one's technical and cognitive limits. ${ }^{27}$ The dual-attending strategy across specialties allows for close professional exchange, open case-based discussions, and reciprocal learning. This can be a win-win situation when both surgeons are at the same skill level, but perhaps more so when each team member has particular strengths from which the other surgeon can learn. Gain in coverage of the operative field can be optimized when strategies are agreed upon, e.g., diagonal positioning of both surgeons and each one commencing the dissection and the placement of instrumentation 
cephalad or caudad, respectively. It was found for complex procedures that a second attending can better anticipate next technical steps. ${ }^{27}$ Familiarity of both surgeons with navigation or freehand techniques for screw placement is required, as simultaneous insertion would be difficult if both surgeons depended on C-arm fluoroscopy or the same navigation tool. ${ }^{13}$ Apart from the measurable advantages a dual-attending strategy may offer, nontechnical aspects of surgery include teamwork and shared decision-making and communication as well, all of which we found positively affected..$^{12} \mathrm{~A}$ further positive implication, which was mentioned in the SRS survey, was reduction of work and stress for each individual surgeon. ${ }^{8}$ In long and complex procedures requiring substantial duration of focus, the dual-attending approach allows for alternating short breaks, necessary to avoid fatigue and keep up high performance-similar to the pilot and copilot situation for long-haul flights. ${ }^{19}$

\section{Implications for Resident and Fellow Training}

Training the next generation of spine surgeons is a key task of academic institutions, and recent data suggest that case numbers have decreased following implementation of government-mandated work-hour restrictions ${ }^{28}$ Residents and fellows are an integral part of the curriculum, and their practical "hands-on" portions of operations may be negatively affected when certain surgical steps are taken out of the learners' hands and performed by another attending. ${ }^{19}$ On the contrary, it has been our experience that case-based discussions and appreciating the manual skills of two attending surgeons with different backgrounds and/or specialties in fact enriches a resident's or fellow's training considerably. Currently, there are no data focusing on how a dual-attending strategy affects the quality of training..$^{21}$ The SRS survey showed that expert opinions are split regarding whether a dual-attending strategy negatively affects the surgical training of residents and/or fellows. ${ }^{15}$ It is clear that hands-on surgical experience should be preserved as much as possible, ${ }^{28}$ but the few carefully selected dual-attending cases (7.5\% of both surgeons' procedures with similar DRG codes) are unlikely to have a strong effect on resident's total case logs and appear not to be optimally suited for junior resident participation. ${ }^{11}$ Having two attendings expeditiously performing the dissection and osteotomies can allow residents to focus on level-specific skills, such as placement of instrumentation or performing decompressions. This is in line with the recent focus on developing surgical zones for resident technical development. Senior residents or fellows may have passed the learning curves for one or several procedural parts, and those residents could be comparable cosurgeons in terms of efficacy and safety. ${ }^{19,29}$ Overall, all surgeons must balance the privilege and responsibility of being educators with their responsibility to their patients. There are numerous spinal procedures for which surgical training was found to be safe, ${ }^{5-9}$ but for ASD correction the safety of surgical training has not yet been ascertained. In our experience, there was resident and/or fellow involvement for each of the 19 dual-attending cases, and a cooperative approach satisfied the expectations of each operating room team member (including the trainees).

\section{Study Strengths and Limitations}

The strengths of this work are that the series comprises all consecutively treated dual-attending cases since the program was initiated, representing valuable experience from a comprehensive, real-life scenario for groups who are interested in establishing similar programs. Moreover, our report includes PROM data and provides a systematic review of the pertinent dual-attending literature for spinal deformity surgery. While prior work on this topic originated from hospitals in which surgeons were paid with salaried wages, ${ }^{21}$ this report provides evidence that a dualattending strategy is also feasible in hospitals with casebased direct financial reimbursement.

The weaknesses of this study are the retrospective design and limited sample size. These features, however, are comparable to those of previously reported studies. . $^{12,13,16-18}$ Because we intended to report our 2-year experience, the case numbers were limited during this time period and no sample size calculations were performed. Our 2-year experience is similar to that for other groups reporting similar research, ${ }^{13,16,17,21}$ while again other groups reported their experience gained after $1,{ }^{18} 3,3,15$ or 7 years. ${ }^{12}$ To compare measurable outcomes of dual-attending cases to those for conventional single-attending cases, we identified two control groups with generally well-matched characteristics (Tables 1 and 2)..$^{14,17}$ Nonsignificant differences in the use of posterior (TLIF) versus lateral (LLIF) interbody devices were also observed in another comparative study by Sethi et al. ${ }^{21}$ Similar to prior works, ${ }^{3,12,18}$ patients in our control group were also treated by contemporary spine attendings, thus minimizing bias introduced by comparing control groups with historical control groups and cases with different surgical strategies, hospital settings, resuscitation and transfusion policies, documentation of complications, etc. ${ }^{12,15,18}$ Importantly, all patients in the control groups were treated by the same pool of complex spine anesthesiologists in a protocoled fashion. It must be pointed out, however, that neither one of the two chosen control groups was perfect, and to study the effects of a dual-attending strategy on the quality of care, patient satisfaction, and cost-effectiveness, a prospective randomized setting would be optimal. Future prospective studies should also address the time taken for various stages of the operation between dual- and single-attending groups. Although our data and the literature review suggest that a dual-attending strategy is beneficial, the value of an experienced resident/fellow or surgical nurse practitioner/ physician assistant should be emphasized. The currently available literature lacks the strength of a randomized prospective trial, originates from selected referral centers, and is often confined to relatively short follow-up times. Therefore, the formulation of definitive and generalizable conclusions regarding the superiority of one strategy over another is not possible.

\section{Future Outlook}

The technical and cognitive limits of individual surgeons and the benefit of receiving help from another surgeon may be difficult to accept for spine surgeons with a more traditional mindset. However, it can be assumed that the number of centers transitioning to dual-attending 
strategies for spinal deformity correction procedures will continue to increase. ${ }^{5,14}$ This is true for academic and highvolume centers seeking to further improve their safety, quality, and outcomes, but perhaps even more so for lowvolume centers where the net gain can be even higher. Removing the barriers imposed by administration, payers, and financial structures is important to ensure broad applicability for the delivery of quality spine care. As the use of dual-surgeon teams increases, the financial ramifications will become even more important.$^{18}$ Defining clinical scenarios in which a second attending surgeon is truly beneficial for both the patient and third-party payers appears important to optimize resource utilization and positively influence reimbursement support.

\section{Conclusions}

Establishing a dual-attending system for ASD correction was feasible and beneficial at our institution. Even though our own institutional results were mostly comparable, dual-attending strategies had a positive safety and outcome profile in the systematic literature review. Further research is needed to define clinical scenarios in which a dual-attending strategy is beneficial not only for patient outcomes but also for cost-effectiveness.

\section{Appendix}

\section{Search Terms Used for the Literature Review}

A: Medline (PubMed) search terms: two[All Fields] AND attending[All Fields] AND dual[All Fields] AND ("surgeons"[MeSH Terms] OR "surgeons"[All Fields] OR "surgeon"[All Fields]) AND ("spine"[MeSH Terms] OR "spine”[All Fields]) AND ("surgery”[Subheading] OR "surgery"[All Fields] OR "surgical procedures, operative"[MeSH Terms] OR ("surgical"[All Fields] AND "procedures"[All Fields] AND “operative"[All Fields]) OR "operative surgical procedures"[All Fields] OR "surgery"[All Fields] OR "general surgery"[MeSH Terms] OR ("general”[All Fields] AND "surgery"[All Fields]) OR "general surgery"[All Fields])

B: SCOPUS search terms: two AND attending AND dual AND surgeon AND spine AND surgery

C: EMBASE search terms: 'two attending dual surgeon spine surgery' OR (two AND attending AND dual AND ('surgeon'/ exp OR surgeon) AND ('spine'/exp OR spine) AND ('surgery'/ exp OR surgery))

D: Web of Science search terms: TOPIC: (two attending dual surgeon spine surgery)

\section{References}

1. Smith JS, Shaffrey CI, Ames CP, Lenke LG. Treatment of adult thoracolumbar spinal deformity: past, present, and future. J Neurosurg Spine. 2019;30(5):551-567.

2. Bridwell KH, Glassman S, Horton W, et al. Does treatment (nonoperative and operative) improve the two-year quality of life in patients with adult symptomatic lumbar scoliosis: a prospective multicenter evidence-based medicine study. Spine (Phila Pa 1976). 2009;34(20):2171-2178.

3. Ames CP, Barry JJ, Keshavarzi S, et al. Perioperative outcomes and complications of pedicle subtraction osteotomy in cases with single versus two attending surgeons. Spine Deform. 2013;1(1):51-58.

4. Smith JS, Klineberg E, Lafage V, et al. Prospective multicenter assessment of perioperative and minimum 2-year postoperative complication rates associated with adult spinal deformity surgery. J Neurosurg Spine. 2016;25(1):1-14.

5. Joswig H, Haile SR, Hildebrandt G, Stienen MN. Residents' learning curve of lumbar transforaminal epidural steroid injections. J Neurol Surg A Cent Eur Neurosurg. 2017;78(5): 460-466.

6. Joswig H, Hock C, Hildebrandt G, et al. Microscopic lumbar spinal stenosis decompression: is surgical education safe? Acta Neurochir (Wien). 2016;158(2):357-366.

7. Stienen MN, Joswig H, Jucker D, et al. Anterior cervical discectomy and fusion: is surgical education safe? Acta Neurochir (Wien). 2015;157(8):1395-1404.

8. Stienen MN, Smoll NR, Hildebrandt G, et al. Early surgical education of residents is safe for microscopic lumbar disc surgery. Acta Neurochir (Wien). 2014;156(6):1205-1214.

9. Stienen MN, Smoll NR, Tessitore E, et al. Surgical resident education in noninstrumented lumbar spine surgery: a prospective observational study with a 4.5 -year follow-up. World Neurosurg. 2015;84(6):1589-1597.

10. Cahill PJ, Pahys JM, Asghar J, et al. The effect of surgeon experience on outcomes of surgery for adolescent idiopathic scoliosis. J Bone Joint Surg Am. 2014;96(16):1333-1339.

11. Heffernan MJ, Seehausen DA, Andras LM, Skaggs DL. Comparison of outcomes after posterior spinal fusion for adolescent idiopathic and neuromuscular scoliosis: does the surgical first assistant's level of training matter? Spine (Phila Pa 1976). 2014;39(8):648-655.

12. Bosch L, Boan C, Falk M, et al. The effect of two attending surgeons on patients with large-curve adolescent idiopathic scoliosis undergoing posterior spinal fusion. Spine Deform. 2017;5:392-395.

13. Chan CY, Kwan MK. Perioperative outcome in posterior spinal fusion for adolescent idiopathic scoliosis: a prospective study comparing single versus two attending surgeons strategy. Spine (Phila Pa 1976). 2016;41(11):E694-E699.

14. Gomez JA, Lafage V, Scuibba DM, et al. Adult scoliosis deformity surgery: comparison of outcomes between one versus two attending surgeons. Spine (Phila Pa 1976). 2017;42(13): 992-998.

15. Halanski MA, Elfman CM, Cassidy JA, et al. Comparing results of posterior spine fusion in patients with AIS: Are two surgeons better than one? J Orthop. 2013;10(2):54-58.

16. Kwan MK, Chan CY. Does a dual attending surgeon strategy confer additional benefit for posterior selective thoracic fusion in Lenke 1 and 2 adolescent idiopathic scoliosis (AIS)? A prospective propensity matching score analysis. Spine J. 2017;17(2):224-229.

17. Kwan MK, Chiu CK, Chan CY. Single vs two attending senior surgeons: assessment of intra-operative blood loss at different surgical stages of posterior spinal fusion surgery in Lenke 1 and 2 adolescent idiopathic scoliosis. Eur Spine $J$. 2017;26(1):155-161.

18. Shrader MW, Wood W, Falk M, et al. The effect of two attending surgeons on the outcomes of posterior spine fusion in children with cerebral palsy. Spine Deform. 2018;6(6): 730-735.

19. Bauer JM, Yanamadala V, Shah SA, Sethi RK. Two surgeon approach for complex spine surgery: rationale, outcome, expectations, and the case for payment reform. $J$ Am Acad Orthop Surg. 2019;27(9):e408-e413.

20. Scheer JK, Sethi RK, Hey LA, et al. Results of the 2015 Scoliosis Research Society survey on single versus dual attending surgeon approach for adult spinal deformity surgery. Spine (Phila Pa 1976). 2017;42(12):932-942.

21. Sethi RK, Pong RP, Leveque JC, et al. The Seattle Spine Team approach to adult deformity surgery: a systems-based approach to perioperative care and subsequent reduction in perioperative complication rates. Spine Deform. 2014;2(2): 95-103. 
22. Stienen MN, Smoll NR, Hildebrandt G, et al. Influence of smoking status at time of surgery for herniated lumbar disk on postoperative pain and health-related quality of life. Clin Neurol Neurosurg. 2014;122:12-19.

23. Kroenke K, Spitzer RL, Williams JB. The Patient Health Questionnaire-2: validity of a two-item depression screener. Med Care. 2003;41(11):1284-1292.

24. Liberati A, Altman DG, Tetzlaff J, et al. The PRISMA statement for reporting systematic reviews and meta-analyses of studies that evaluate healthcare interventions: explanation and elaboration. BMJ. 2009;339:b2700.

25. McCarthy I, O'Brien M, Ames C, et al. Incremental costeffectiveness of adult spinal deformity surgery: observed quality-adjusted life years with surgery compared with predicted quality-adjusted life years without surgery. Neurosurg Focus. 2014;36(5):E3.

26. Uribe JS, Deukmedjian AR, Mummaneni PV, et al. Complications in adult spinal deformity surgery: an analysis of minimally invasive, hybrid, and open surgical techniques. Neurosurg Focus. 2014;36(5):E15.

27. Novick RJ, Lingard L, Cristancho SM. The call, the save, and the threat: understanding expert help-seeking behavior during nonroutine operative scenarios. J Surg Educ. 2015;72(2): 302-309.

28. Stienen MN, Bartek J Jr, Czabanka MA, et al. Neurosurgical procedures performed during residency in Europe-preliminary numbers and time trends. Acta Neurochir (Wien). 2019; 161(5):843-853.
29. Auerbach JD, Lonner BS, Antonacci MD, Kean KE. Perioperative outcomes and complications related to teaching residents and fellows in scoliosis surgery. Spine (Phila Pa 1976). 2008;33(10):1113-1118.

\section{Disclosures}

Dr. Cheng reports receiving royalties from NuVasive, Globus Medical, SpineCraft, and Spine Wave and having direct stock ownership in Cytonics, Spine Innovations, SpinalCyte, and Notogen. Dr. Veeravagu reports being a consultant for NuVasive, Medtronic, and Surgical Theater.

\section{Author Contributions}

Conception and design: Stienen, Cheng, Veeravagu. Acquisition of data: Stienen. Analysis and interpretation of data: Stienen, Cheng, Veeravagu. Drafting the article: Stienen. Critically revising the article: Cheng, Medress, Varshneya, Ho, Ratliff, Veeravagu. Reviewed submitted version of manuscript: Cheng, Medress, Varshneya, Ho, Ratliff, Veeravagu. Approved the final version of the manuscript on behalf of all authors: Stienen. Statistical analysis: Stienen. Administrative/technical/material support: Cheng, Ratliff, Veeravagu. Study supervision: Cheng, Ratliff, Veeravagu.

\section{Correspondence}

Martin N. Stienen: Stanford University, Stanford, CA. mnstienen@gmail.com. 